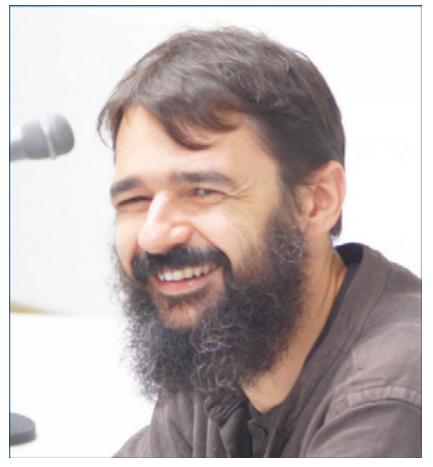

DIKAIOS SAKELLARIOU, PhD

Dikaios Sakellariou, $\mathrm{PhD}$, is a Reader at the School of Healthcare Sciences at Cardiff University, UK. He is a disability researcher, interested in experiences of disability, health inequalities, and access to cancer services for people with disabilities. His most recent book is Disability, Normalcy, and the Everyday (2018), co-edited with Gareth Thomas. His research on access to cancer services for people with disabilities has been supported by Tenovus Cancer Care.

doi: 10.6004/jnccn.2020.7614

The ideas and viewpoints expressed in this commentary are those of the author and do not necessarily represent any policy, position, or program of NCCN.

\section{Cancer Disparities for People With Disabilities: Bridging the Gap}

\author{
Dikaios Sakellariou, PhD, and Elena S. Rotarou, PhD
}

$T_{\text {tata }}$ There are approximately one billion people with disabilities globally. Despite the fact that disability affects roughly $15 \%$ of the global population, it is often overlooked both in health policy and in research on health access. ${ }^{1}$ Therefore, not only do people with disabilities experience disparities in health access and outcomes, but their needs and experiences also remain invisible. ${ }^{2}$ In this issue of $J N C C N$, lezzoni et $a^{3}$ draw attention to this population, exploring the association between disability and cancer in the United States. This is one of the few studies that focus specifically on examining incidence of cancer among people with disabilities, a particularly important yet underexplored issue. Although there is a growing body of evidence on disparities in cancer service utilization for people with disabilities, predominantly screening and diagnostic services, limited information is available on cancer incidence.

People with disabilities are among the most disadvantaged population groups; they often experience structural disadvantage in the form of increased poverty levels, lower literacy levels, and higher rates of unemployment or informal employment compared with the general population. ${ }^{4}$ Such structural disadvantage intersects with "ableism," which is discrimination against people with disabilities and the expectation that all bodies can or ought to conform to an idealised nondisabled norm. This intersection leads to compromised access to healthcare services and to living conditions linked to poorer health. Direct and indirect discrimination against people with disabilities can lead to increased barriers to accessing healthcare and reduced use of cancer services.

An increasing body of evidence indicates lower participation in cancer screening for people with disabilities, due to a number of physical, institutional, financial, and attitudinal barriers. Such barriers include inaccessible healthcare facilities and/or equipment, lack of social support, financial constraints, past negative experiences with healthcare professionals, discomfort, inadequate information, lack of or inappropriate transport, and inadequate training of healthcare professionals, among other factors. 5,6 Similar sets of barriers and patterns of disparities are observed globally across different healthcare systems; evidence suggests that people with disabilities experience worse access to cancer screening not only in the United States ${ }^{7}$ but also across the world, including even in countries with universal health coverage and public healthcare that is free at the point of delivery, such as the United Kingdom. ${ }^{8}$

Various studies also underline the existence of disparities in cancer treatment and substandard experiences of service provision..$^{9,10}$ Behind the statistics of cancer screening underuse are human stories: men and women with disabilities who-worried about new, unfamiliar symptoms-try to get screened but whom doctors are reluctant to refer, possibly due to diagnostic overshadowing; wheelchair users being asked to stand up; and healthcare professionals not exploring ways to adapt processes to make them disability-inclusive, exemplifying a general lack of disability inclusiveness in the provision of healthcare services. ${ }^{10,11}$

See page 1031 for related article. 
The study by lezzoni et $\mathrm{al}^{3}$ provides novel insights and much-needed knowledge about the associations between disability and cancer incidence in the United States. The researchers used a nationally representative survey-the 2010-2017 National Health Interview Surveys-to investigate the relationship between disability and four common types of cancer: colorectal, prostate, ovarian, and non-Hodgkin's lymphoma. The study included people who had movement difficulties or complex activity limitations that predated the cancer diagnosis. In line with existing evidence, lezzoni et al ${ }^{3}$ found that people with disabilities were older, had lower education levels, were generally poorer, had a higher body mass index, and were more likely to have a smoking history. Importantly, study findings showed that people with preexisting disabilities had significantly higher rates of cancer and that they were older when initially diagnosed compared with people without disability. Multivariable regressions, accounting for sociodemographic and risk factor characteristics, showed that preexisting disability-disability that predated the cancer diagnosis and was not a result of cancer-was strongly associated with colorectal and prostate cancers, and non-Hodgkin's lymphoma.

The study findings may be alarming, but they are also unsurprising considering the barriers that people with disabilities experience when seeking screening services. The study should serve as a call to action for policymakers, healthcare managers, and clinicians. Even when people with disabilities do access these services, diagnostic overshadowing, which is the erroneous attribution of new symptoms to a patient's preexisting disability, may delay cancer diagnosis. The study underlines the need to ensure the provision of appropriate screening and comprehensive exploration of all new signs and symptoms for people with disabilities, in order to ensure timely diagnosis.

The evidence provided by lezzoni et $\mathrm{al}^{3}$ adds real urgency to the need to work toward reducing cancer disparities for people with disabilities, through taking a twinforked approach, focusing both on the provision of disability-inclusive healthcare, including cancer services, and on the exploration and reduction of increased cancer incidence. It is important to disaggregate existing cancer data by disability in order to understand the mechanisms associated with cancer disparities for people with disabilities. Although a wealth of cancer-related evidence is available, databases often do not include information on disability, making it very difficult to measure disability-based disparities. The invisibility of this population in cancer statistics might contribute to their needs being overlooked. Ensuring that all cancer data include the Washington Group questions, ${ }^{12}$ which aim to identify people with disabilities in datasets, can provide visibility for this population in cancer statistics. This would allow an in-depth exploration of the interactions between factors such as age, sex, disability, and illness and environmental influences, including geographic location, government policies, and the natural and built environment, in order to better understand disparities in cancer screening, incidence, stage at diagnosis, treatment, and outcomes for people with disabilities.

Making disability-disaggregated data available is one step toward bridging cancer disparities for people with disabilities. This needs to be coupled with an effort to make all health services disability-inclusive, because the mere existence of services does not guarantee accessibility and usability. To ensure that cancer services are disability-inclusive and ultimately accessible, they must be available, affordable, relevant, appropriate, and acceptable to service users with disabilities. All of these dimensions of accessibility are equally important, and they interact with each other to facilitate or inhibit access to services. Although the Americans with Disabilities Act in the United States and similar pieces of legislation across the world, including the United Nations Convention on the Rights of Persons with Disabilities, explicitly prohibit disability-based discrimination in healthcare, people with disabilities face

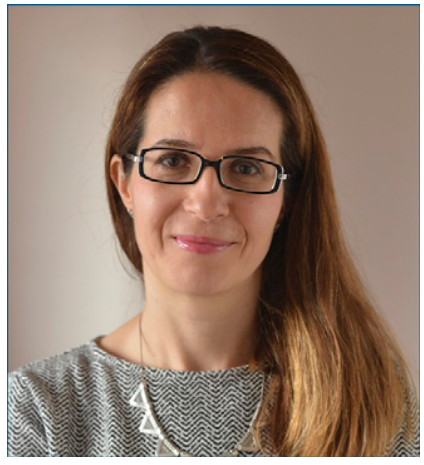

ELENA S. ROTAROU, PhD

Elena S. Rotarou, PhD, is an Assistant Professor at the Department of Public Health, Faculty of Medicine and Science at the Universidad San Sebastián, Chile. She is an interdisciplinary researcher in public health, combining various disciplines, such as health economics, social epidemiology, and economic development. She has published extensively in the areas of health inequalities, health systems evaluation, and health policies. 
several barriers when they seek to access services. These barriers must be identified and addressed.

It is important that policymakers work toward inclusive cancer care provision through the introduction of public health campaigns that promote inclusive preventive services. Health professionals need to receive appropriate education and training regarding disability in order to dispel assumptions and negative attitudes. ${ }^{13}$ Listening to people with disabilities who have cancer is one of the most effective ways to change practice and create inclusive cancer services. Furthermore, better communication among various professionals and across different teams involved in a patient's care is paramount and can help raise awareness of how disability can interact with cancer-related care. At the same time, the creation of more accessible environments is crucial, including screening equipment such as that used for mammograms, and treatment processes such as radiotherapy.
As the population ages and an increasing number of people live with cancer and disability, ensuring that cancer services are disability-inclusive is more imperative than ever. Early and fast diagnosis, equity of access to treatment and care, and inclusive health promotion campaigns are key for the development of healthcare services that better respond to the needs of people with disabilities. Improving cancer outcomes is a complex endeavour that cannot be realized without the explicit consideration of people with disabilities and their participation in the decision-making processes.

Disclosures: The authors have disclosed that they have no financial interests, arrangements, affiliations, or commercial interests with the manufacturers of any products discussed in this article or their competitors.

Correspondence: Dikaios Sakellariou, PhD, Cardiff University, School of Healthcare Sciences Eastgate House, Newport Road 35-43, Cardiff, CF24 OAB, UK. Email: sakellarioud@cardiff.ac.uk

\section{References}

1. Kuper H, Heydt P. The Missing Billion: Access to Health Services for 1 Billion People with Disabilities. London, England: London School of Hygiene and Tropical Medicine; 2019.

2. Krahn GL, Walker DK, Correa-De-Araujo R. Persons with disabilities as an unrecognized health disparity population. Am J Public Health 2015; 105(S2):S198-206.

3. lezzoni LI, Rao SR, Agaronnik N, El-Jawahri A. Cross-sectional analysis of the associations between four common cancers and disability. J Natl Compr Canc Netw 2020;18:1031-1044

4. World Health Organization. World Report on Disability. Accessed July 10, 2020. Available at: https://www.who.int/disabilities/world_report/2011/ report.pdf?ua $=1$

5. Edwards DJ, Sakellariou D, Anstey S. Barriers to, and facilitators of, access to cancer services and experiences of cancer care for adults with a physical disability: a mixed methods systematic review [published online October 11, 2019]. Disabil Health J, doi: 10.1016/j.dhjo.2019.100844

6. Merten JW, Pomeranz JL, King JL, et al. Barriers to cancer screening for people with disabilities: a literature review. Disabil Health J 2015; 8:9-16.
7. Steele CB, Townsend JS, Courtney-Long EA, Young M. Prevalence of cancer screening among adults with disabilities, United States, 2013. Prev Chronic Dis 2017;14:E09.

8. Floud S, Barnes I, Verfürden M, et al. Disability and participation in breast and bowel cancer screening in England: a large prospective study. $\mathrm{Br} \mathrm{J}$ Cancer 2017;117:1711-1714.

9. lezzoni LI, Ngo LH, Li D, et al. Treatment disparities for disabled Medicare beneficiaries with stage I non-small cell lung cancer. Arch Phys Med Rehab 2008;89:595-601.

10. Sakellariou D, Anstey S, Polack $S$, et al. Pathways of disability-based discrimination in cancer care [published online July 31, 2019]. Crit Public Health, doi: 10.1080/09581596.2019.1648762

11. Sakellariou D, Anstey S, Gaze S, et al. Barriers to accessing cancer services for adults with physical disabilities in England and Wales: an interviewbased study. BMJ Open 2019;9:e027555.

12. Groce NE, Mont D. Counting disability: emerging consensus on the Washington Group questionnaire. Lancet Glob Health 2017;5:e649-650.

13. Shakespeare T, lezzoni LI, Groce N. Disability and the training of health professionals. Lancet 2009;374:1815-1816. 